\title{
Determination of the effective constitutive parameters of bianisotropic metamaterials from reflection and transmission coefficients
}

\author{
Zhaofeng Li, ${ }^{*}$ Koray Aydin, and Ekmel Ozbay \\ Nanotechnology Research Center, Department of Physics, and Department of Electrical and Electronics Engineering, \\ Bilkent University, Bilkent, 06800 Ankara, Turkey
}

(Received 5 November 2008; published 25 February 2009)

\begin{abstract}
We propose a method to retrieve the effective constitutive parameters of a slab of bianisotropic metamaterial from reflection and transmission coefficients (or scattering parameters). In our retrieval method, only the scattering parameters in one propagation direction are used. Analytical inversion equations are derived in order to retrieve the effective parameters of the permittivity, permeability, and magnetoelectric coupling coefficient of the bianisotropic metamaterial. To demonstrate the validity of the method, we used it to retrieve the parameters of four different metamaterials, among which two were normal media without bianisotropy and the other two were bianisotropic media. In using our retrieval method, including bianisotropy, the intrinsic differences between a normal medium and a bianisotropic medium were illustrated clearly. Our simulation and retrieval results also corroborate the conclusions of the previously published literature.
\end{abstract}

DOI: 10.1103/PhysRevE.79.026610

PACS number(s): 41.20.Jb, 42.25.Bs

\section{INTRODUCTION}

In 1968, Veselago developed the concept of a material with a negative refractive index, which simultaneously exhibits negative permittivity $\varepsilon$ and negative permeability $\mu$ [1]. For plane waves propagating in such materials, the electrical field $E$, magnetic field $H$, and wave vector $k$ follow the left-hand rule and this gives rise to the name left-handed materials (LHMs). However, in the following three decades, no more important progress was made because such materials with a negative refractive index do not exist naturally. It was not until Pendry et al. proposed two composite components for the construction of such materials that the topic of LHMs was revived. One component is composed of the arrays of thin metal wires, which give negative permittivity [2]. The other component is composed of arrays of split-ring resonators (SRRs) that provide for negative permeability [3]. By combining these two components, a metamaterial with a negative refractive index can be obtained within a certain frequency range [4]. It has been proposed that these metamaterials respond to electromagnetic radiation as continuous materials when the wavelength is much larger than the spacing between the composite components and the size of these respective components. Therefore, it is reasonable to assign values of permittivity $\varepsilon$ and permeability $\mu$ for a metamaterial. In order to seek such effective constitutive parameters, there are three methods. One method is to numerically calculate the ratios of the electromagnetic field in the metamaterial $[2,5]$. This is easy for numerical simulations but rather difficult in experimental situations. Another method is to estimate the effective constitutive parameters by approximate analytical models [6]. Although this method can illuminate the physical properties from the geometrical structures of the composite components, it is not easy to deal with complex structures. Apart from the above two methods, Smith et al. proposed a method to retrieve the constitutive parameters by

\footnotetext{
*zhaofengli@bilkent.edu.tr
}

using scattering parameters ( $S$ parameters) [7], which is suitable to be applied for both numerical simulation and experimental measurements. Several reports have been published so far discussing the applications of the retrieval method under varied situations [8-11]; most of them deal with the isotropic parameters of permittivity and permeability. However, it has already been demonstrated that most metamaterials are intrinsically anisotropic due to the asymmetry of the composite components such as split-ring resonators $[6,12]$. For a bianisotropic metamaterial, the propagation wave vector is related not only to permittivity and permeability but also to the magnetoelectric coupling coefficient. Moreover, there are some special metamaterial designs that take advantage of the property of bianisotropy [13]. Consequently, in order to obtain a true picture for these metamaterials, it is important to present not only the effective parameters of permittivity and permeability but also some other important parameters, such as the electromagnetic coupling coefficient as presented in the retrieval results. Only then one can provide true data for the effective refractive index. Actually, there is an early report where the authors attempt to retrieve the constitutive parameters of a bianisotropic metamaterial by considering the $S$ parameters in three orthogonal directions [14]. Compared to the retrieval method for isotropic materials, this method for bianisotropic materials is quite complex. Here, we will demonstrate that, by considering the $S$ parameters in only one direction, it is sufficient to retrieve the constitutive parameters for bianisotropic metamaterials. The following sections are arranged as follows. In Sec. II, we provide the expressions for the constitutive parameters for a metamaterial composed of SRRs. Based on the constitutive parameters of the bianisotropic material, we deduce the formulas for the calculation of the $S$ parameters. Then we invert these formulas in order to obtain the analytical expressions that can be used to calculate the constitutive parameters from the $S$ parameters. In Sec. III, we apply our analytical inversion expression to retrieve the constitutive parameters of a series of composite metamaterials. Some related discussions are also presented. The conclusions are presented in Sec. IV. 


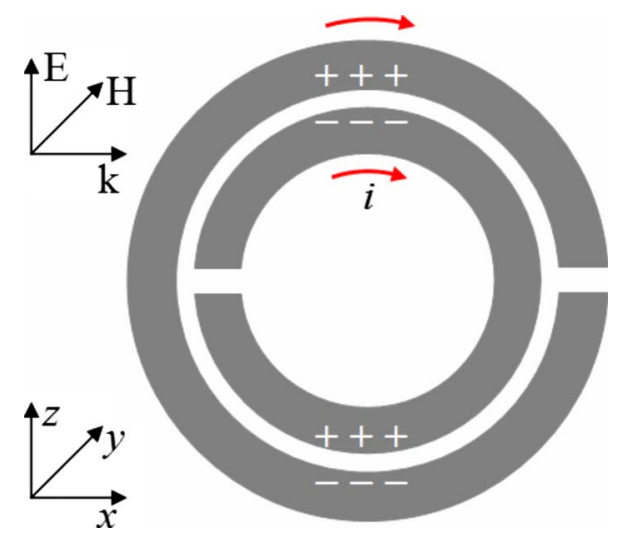

FIG. 1. (Color online) Schematic of a split-ring resonator used to construct metamaterials. When a plane wave polarized along the $z$ axis is incident in the $x$ direction, the metamaterial will show bianisotropy.

\section{RETRIEVAL METHOD}

\section{A. The constitutive parameters of a bianisotropic metamaterial}

Figure 1 shows a schematic of a commonly used edgecoupled SRR. The SRR structure consists of two concentric metallic rings that are both interrupted by a small gap. When a plane wave is incident in the $x$ direction with an electrical field in the $z$ direction and a magnetic field in the $y$ direction, the SRR will respond with a bianisotropic property. This is because the electrical field in the $z$ direction can induce a magnetic dipole in the $y$ direction due to the asymmetry of the inner and outer rings, while the magnetic field in the $y$ direction can also induce an electrical dipole in the $z$ direction. By assuming that the medium is reciprocal [15] and that the harmonic time dependence is $e^{-i \omega t}$, we can write the constitutive relationships as

$$
\begin{aligned}
& \vec{D}=\overline{\bar{\varepsilon}} \cdot \vec{E}+\overline{\bar{\xi}} \cdot \vec{H}, \\
& \vec{B}=\overline{\bar{\mu}} \cdot \vec{H}+\overline{\bar{\zeta}} \cdot \vec{E},
\end{aligned}
$$

where

$$
\begin{gathered}
\overline{\bar{\varepsilon}}=\varepsilon_{0}\left(\begin{array}{ccc}
\varepsilon_{x} & 0 & 0 \\
0 & \varepsilon_{y} & 0 \\
0 & 0 & \varepsilon_{z}
\end{array}\right), \quad \overline{\bar{\mu}}=\mu_{0}\left(\begin{array}{ccc}
\mu_{x} & 0 & 0 \\
0 & \mu_{y} & 0 \\
0 & 0 & \mu_{z}
\end{array}\right), \\
\overline{\bar{\xi}}=\frac{1}{c}\left(\begin{array}{ccc}
0 & 0 & 0 \\
0 & 0 & 0 \\
0 & -i \xi_{0} & 0
\end{array}\right), \quad \overline{\bar{\zeta}}=\frac{1}{c}\left(\begin{array}{ccc}
0 & 0 & 0 \\
0 & 0 & i \xi_{0} \\
0 & 0 & 0
\end{array}\right) .
\end{gathered}
$$

Here, $\varepsilon_{0}$ and $\mu_{0}$ are the permittivity and permeability of the vacuum, respectively, and $c$ is the speed of light in vacuum. The seven unknowns $\varepsilon_{x}, \varepsilon_{y}, \varepsilon_{z}, \mu_{x}, \mu_{y}, \mu_{z}$, and $\xi_{0}$ are quantities without dimensions. When a plane wave that is polarized in the $z$ direction is incident in the $x$ direction, three parameters $\left(\varepsilon_{z}, \mu_{y}\right.$, and $\left.\xi_{0}\right)$ will be active, while the other four parameters $\left(\varepsilon_{x}, \varepsilon_{y}, \mu_{x}\right.$, and $\left.\mu_{z}\right)$ will not be involved in the bianisotropic process and, therefore, are out of the scope

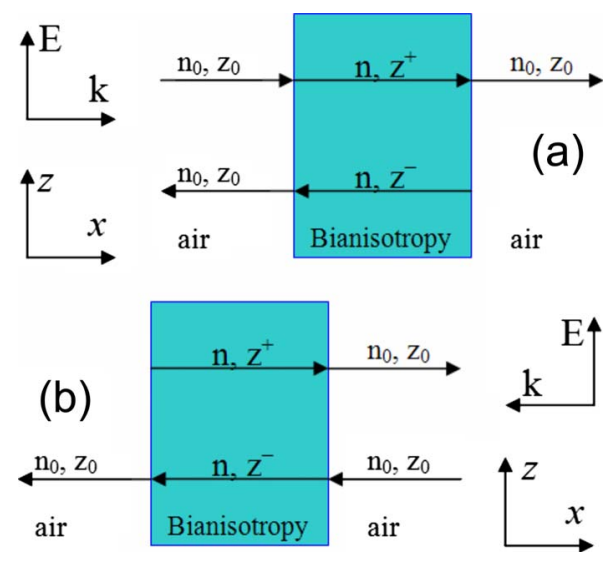

FIG. 2. (Color online) Schematics of a homogeneous bianisotropic slab placed in open space for the calculation of $S$ parameters. (a) and (b) are for plane waves incident in the $+x$ and $-x$ directions, respectively.

of the present study. According to the formulas in Ref. [15], one can easily obtain the expressions for the effective constitutive parameters based on $\varepsilon_{z}, \mu_{y}$, and $\xi_{0}$. However, there is one thing that one should pay attention to. Compared to an isotropic material, the most interesting and important feature of a bianisotropic material is that the characteristic impedances have different values for the waves propagating in the two opposite directions of the $x$ axis. For an electromagnetic (EM) wave traveling in the $\pm x$ direction, the impedances will be

$$
z^{+}=\frac{\mu_{y}}{n+i \xi_{0}}, \quad z^{-}=\frac{\mu_{y}}{n-i \xi_{0}},
$$

respectively. Here $n$ is the effective refractive index, which has the same value for the EM wave traveling in the two opposite directions on the $x$ axis,

$$
n= \pm \sqrt{\varepsilon_{z} \mu_{y}-\xi_{0}^{2}}
$$

\section{B. The retrieval formulas}

As aforementioned in the Introduction, a periodic metamaterial can be approximated as a homogeneous medium under the condition of a long wavelength. Therefore, it is here that we use a simplified model of a homogeneous bianisotropic material slab for the calculation of the $S$ parameters. Figure 2 shows the schematics of a homogeneous bianisotropic material slab that is placed in an open space. There are two different situations to be considered, i.e., incidence in the $+x$ and $-x$ directions. After applying the boundary continuous conditions, it is easy to obtain the expressions for the $S$ parameters by using the transfer matrix method [15]. When the incidence is in the $+x$ direction as shown in Fig. 2(a), the corresponding reflection $\left(S_{11}\right)$ and transmission $\left(S_{21}\right)$ coefficients are as

$$
S_{11}=\frac{2 i \sin \left(n k_{0} l\right)\left[n^{2}+\left(\xi_{0}+i \mu_{y}\right)^{2}\right]}{\left[\left(\mu_{y}+n\right)^{2}+\xi_{0}^{2}\right] e^{-i n k_{0} l}-\left[\left(\mu_{y}-n\right)^{2}+\xi_{0}^{2}\right] e^{i n k_{0} l}},
$$




$$
S_{21}=\frac{4 \mu_{y} n}{\left[\left(\mu_{y}+n\right)^{2}+\xi_{0}^{2}\right] e^{-i n k_{0} l}-\left[\left(\mu_{y}-n\right)^{2}+\xi_{0}^{2}\right] e^{i n k_{0} l}},
$$

where $l$ is the thickness of the homogeneous bianisotropic material slab and $k_{0}$ is the wave number of light in free space. For the case when the incidence is in the $-x$ direction, as shown in Fig. 2(b), we obtain the corresponding reflection $\left(S_{22}\right)$ and transmission $\left(S_{12}\right)$ coefficients as

$$
\begin{aligned}
& S_{12}=\frac{4 \mu_{y} n}{\left[\left(\mu_{y}+n\right)^{2}+\xi_{0}^{2}\right] e^{-i n k_{0} l}-\left[\left(\mu_{y}-n\right)^{2}+\xi_{0}^{2}\right] e^{i n k_{0} l}}, \\
& S_{22}=\frac{2 i \sin \left(n k_{0} l\right)\left[n^{2}+\left(\xi_{0}-i \mu_{y}\right)^{2}\right]}{\left[\left(\mu_{y}+n\right)^{2}+\xi_{0}^{2}\right] e^{-i n k_{0} l}-\left[\left(\mu_{y}-n\right)^{2}+\xi_{0}^{2}\right] e^{i n k_{0} l}} .
\end{aligned}
$$

It is clearly seen that $S_{21}$ is equal to $S_{12}$, but $S_{11}$ is not equal to $S_{22}$. Therefore, we have three independent equations to solve here for the three unknowns $\left(n, \mu_{y}\right.$, and $\left.\xi_{0}\right)$. After some trivial treatment, we first obtain the analytical expression for the refractive index $n$, which is

$$
\cos \left(n k_{0} l\right)=\frac{1-S_{11} S_{22}+S_{21}^{2}}{2 S_{21}} .
$$

Obviously, when $S_{11}$ is equal to $S_{22}$, Eq. (9) will degenerate into a standard retrieval equation [7]. When solving for $n$ from Eq. (9), one must determine one branch from many branches of solutions. Fortunately, there have been several reports $[7,11]$ dealing with this problem. Therefore, we will not detail it here. For a passive medium, the solved $n$ must obey the condition

$$
n^{\prime \prime} \geqslant 0
$$

where $(\cdot)^{\prime \prime}$ denotes the imaginary part operator. After $n$ is obtained, other constitutive parameters can be obtained easily:

$$
\begin{gathered}
\xi_{0}=\left(\frac{n}{-2 \sin \left(n k_{0} l\right)}\right)\left(\frac{S_{11}-S_{22}}{S_{21}}\right), \\
\mu_{y}=\left(\frac{i n}{\sin \left(n k_{0} l\right)}\right)\left(\frac{2+S_{11}+S_{22}}{2 S_{21}}-\cos \left(n k_{0} l\right)\right), \\
\varepsilon_{z}=\frac{\left(n^{2}+\xi_{0}^{2}\right)}{\mu_{y}} .
\end{gathered}
$$

Consequently, the impedances $\left(z^{+}\right.$and $\left.z^{-}\right)$can be obtained from Eq. (3). Again, for a passive medium, the following conditions should be satisfied:

$$
z^{+\prime} \geqslant 0, \quad z^{-\prime} \geqslant 0,
$$

where $(\cdot)^{\prime}$ denotes the real part operator. So far, all of the constitutive parameters that are related to bianisotropy are retrieved.

It might be helpful to compare our retrieval method to the one proposed in Ref. [14] previously. In the retrieval method of Ref. [14], six directions of incidence are needed in three orthogonal directions. This will yield twelve equations for seven unknowns to be solved. Among these unknowns, five

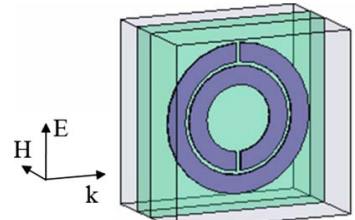

(a)

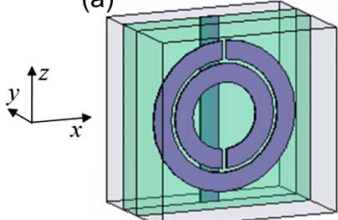

(c)

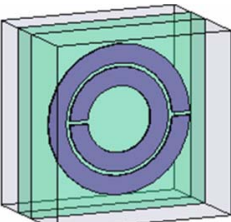

(b)

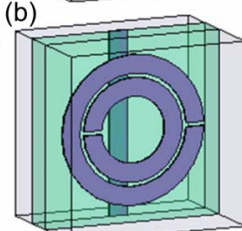

(d) (e)

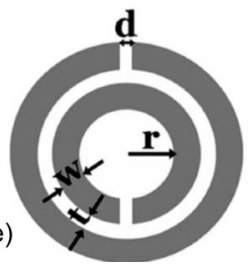

FIG. 3. (Color online) (a)-(d) are the schematics of four single cells of metamaterials of SRR-I, SRR-II, CMM-I, and CMM-II, respectively. (e) shows the detailed structure of the SRR included in the metamaterials. The geometric parameters of the SRR are $d=t$ $=0.2 \mathrm{~mm}, w=0.9 \mathrm{~mm}$, and $r=1.6 \mathrm{~mm}$.

are therefore solved twice in this overdetermined problem. This may become quite complicated especially in experimental situations. Now, in our technique, only two directions of incidence are needed in one direction. One only needs to solve the three equations obtained for three unknowns. Consequently, our retrieval method greatly improves the retrieval efficiency for both theoretical and experimental studies of metamaterials.

\section{RETRIEVAL FOR COMPOSITE METAMATERIALS}

Our retrieval method can be used to retrieve both lossy and lossless metamaterials. In fact, there is no significant difference between the retrieval processes of these two types of metamaterials by using our method. Considering that most metamaterials that are studied nowadays are lossy media in practice, here we will only demonstrate the retrieval results for lossy metamaterials for the purpose of conciseness.

Figure 3 shows four single cells of the metamaterials under study. The incident wave is a plane wave with its wave vector $k$ in the $x$ direction and the $E$ field polarized in the $z$ direction. Figure 3(a) is a metamaterial that is composed of a pure SRR with its gap opened in the $z$ direction. This metamaterial is denoted as SRR-I, and should not show bianisotropy for the present incidence. Figure $3(\mathrm{~b})$ is a metamaterial that is composed of a pure SRR with its gap opened in the $x$ direction. This metamaterial is denoted as SRR-II, and should show bianisotropy for the present incidence. By adding infinite wires into the structure of Fig. 3(a), a composite metamaterial (CMM) is constructed as shown in Fig. 3(c). This composite metamaterial is denoted as CMM-I, and should have a negative index within a certain frequency range, but no bianisotropy. Similarly, Fig. 3(d) shows another 


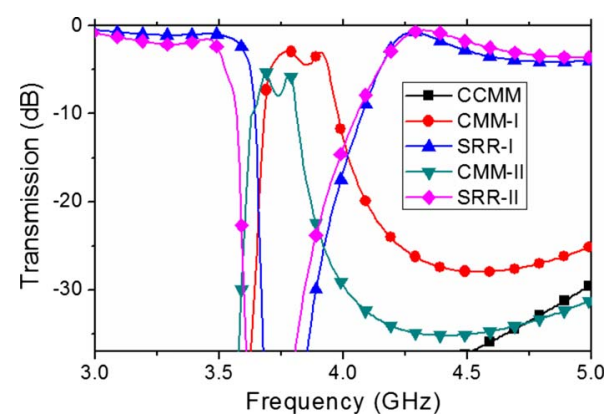

FIG. 4. (Color online) Transmission spectra of the metamaterials SRR-I, SRR-II, CMM-I, CMM-II, and CCMM.

composite metamaterial that is constructed by adding infinite wires into the structure of Fig. 3(b). This composite metamaterial is denoted as CMM-II, and should show bianisotropy and a possible negative index within a certain frequency range. In order to illustrate the SRR structure more clearly, we show it in Fig. 3(e). The design and dimensions of the SRR are the same as those of Ref. [16]. A CMM consisting of SRRs and wires is fabricated using a conventional printed circuit board process with 30- $\mu$ m-thick copper patterns on both sides of an FR-4 dielectric substrate. The FR-4 board has a thickness of $1.6 \mathrm{~mm}$. The dielectric constant is 4.4 and the conductance is $0.0068 \mathrm{~S} / \mathrm{m}$. The geometric parameters of the SRR are $d=t=0.2 \mathrm{~mm}, w=0.9 \mathrm{~mm}$, and $r=1.6 \mathrm{~mm}$. The width of the infinite straight wires is $0.9 \mathrm{~mm}$. The dimensions of a unit cell are $a_{x}=a_{y}=8.8 \mathrm{~mm}$ and $a_{z}=6.5 \mathrm{~mm}$.

Before we begin to demonstrate the retrieval results, we would like to calculate the transmissions for all the above four metamaterials. As a reference, we also calculate the transmission spectrum for one more metamaterial which is composed of infinite wires and closed SRRs [16], and this metamaterial is denoted as closed CMM (CCMM). If a pass band in the transmission spectrum of the CMM medium lies within the forbidden band of its corresponding CCMM medium, this pass band is then considered to be a band with a negative refractive index $[16,17]$. In the calculations, the metamaterials have a thickness of four cells in the wave propagation direction ( $x$ direction), and periodic boundary conditions are set for the other two orthogonal directions. Figure 4 shows the calculation results. It can be clearly seen that there is a pass band in the transmission spectrum of the CMM-I metamaterial, which falls in the stop bands of the corresponding metamaterials SRR-I and CCMM. This pass band is usually considered as a band with a negative index. Similar results can be found for CMM-II when compared with SRR-II and CCMM. However, there are still some differences between the results of SRR(CMM)-I and SRR(CMM)-II. Compared to the stop band in the transmission spectrum of SRR-I, the stop band of SRR-II is clearly wider at the lower edge. This phenomenon corroborates the experimental and simulation results that have been reported previously [12]. This phenomenon was thought to be qualitative evidence for the existence of bianisotropy in the metamaterial SRR-II. On the other hand, compared to the pass band in the transmission spectrum of CMM-I, the pass band of CMM-II is evidently narrower and, moreover, it is shifted to the lower-frequency end. We will illustrate later (a)
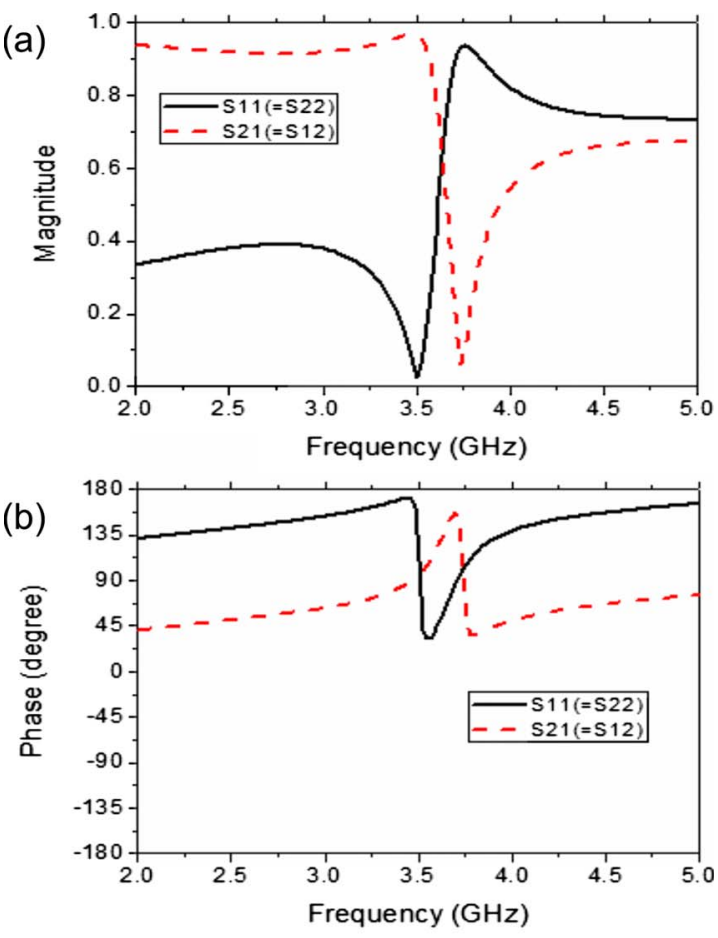

(c)
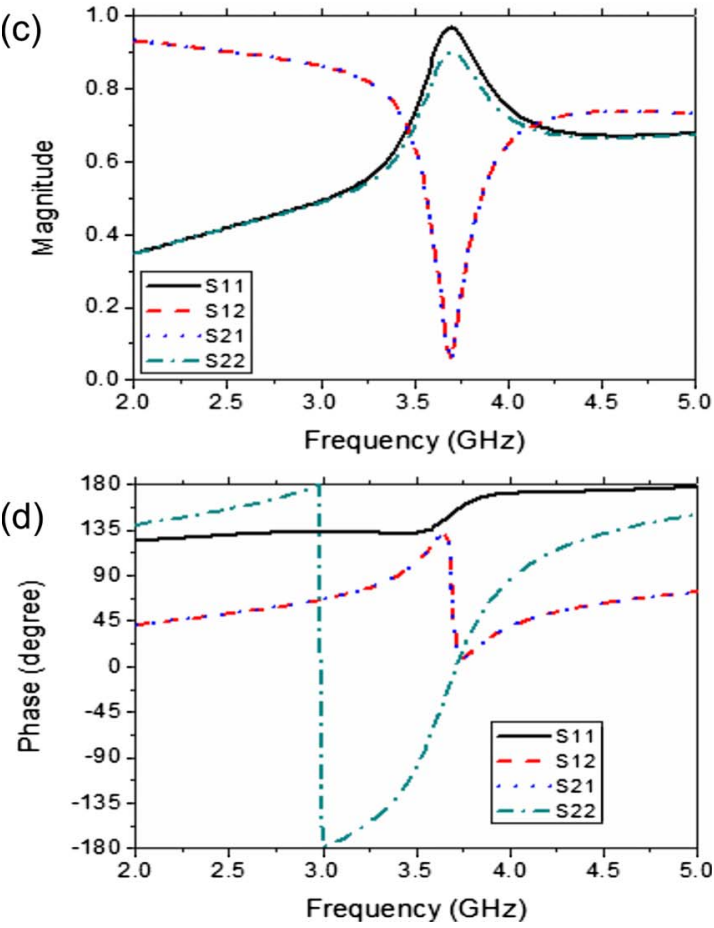

FIG. 5. (Color online) (a) Magnitude and (b) phase of the calculated $S$ parameters for the unit cell of SRR-I in Fig. 3(a). (c) Magnitude and (d) phase of the calculated $S$ parameters for the unit cell of SRR-II in Fig. 3(b).

that this phenomenon of a narrower pass band actually also results from the bianisotropy.

\section{A. Metamaterials composed of SRRs}

Figures 5(a)-5(d), show the amplitude and phase information of the calculated $S$ parameters for the metamaterials 
SRR-I and SRR-II, respectively. It can be seen that for the SRR-I metamaterial $S_{11}$ is equal to $S_{22}$, and $S_{12}$ is equal to $S_{21}$, since the structure is symmetric in the $x$ direction. Accordingly, using the standard retrieval method [7] or our method considering bianisotropy [Eq. (9)], the retrieval results for an effective refractive index will be the same. For the SRR-II metamaterial, $S_{12}$ is equal to $S_{21}$, but neither the amplitude nor the phase of $S_{11}$ is equal to that of $S_{22}$. In this case, the retrieval results for the effective refractive index will be different when using the standard retrieval method or our method. This is an important reason as to why bianisotropy should be included in the retrieval process.

Based on all the above data, we retrieve the constitutive parameters for the metamaterials SRR-I and SRR-II; the results are shown in Fig. 6. Figure 6(a) shows the retrieval results for the effective index. Figure 6(a) shows the effective indices of the two metamaterials, which are quite similar except for the fact that the curve of SRR-II has a redshift in the frequency range below $3.8 \mathrm{GHz}$. Figure 6(b) is the retrieval results for the effective impedances. Obviously, SRR-I has a uniform impedance, while SRR-II has two different impedances for two opposite directions on the $x$ axis. After comparing the $z^{\prime}$ curve of SRR-I and $z^{+\prime}$ curve of SRR-II, one finds that, in the regions where the real part of the impedance is near to zero, SRR-II is wider than SRR-I at the lower edge. This explains why SRR-II has a wider stop band than SRR-I, as shown in Fig. 4.

Now, let us check the effective parameters of $\varepsilon_{z}, \mu_{y}$, and $\xi_{0}$ that are shown in Figs. 6(c)-6(e). For $\varepsilon_{z}$, the most significant difference is that the $\varepsilon_{z}{ }^{\prime}$ of SRR-I shows antiresonant behavior [8] while the $\varepsilon_{z}{ }^{\prime}$ of SRR-II shows normal resonant behavior. For $\mu_{y}{ }^{\prime}$, one can clearly see that the curve of SRR-II has a redshift compared to that of SRR-I. Moreover, the band of negative $\mu_{y}{ }^{\prime}$ of SRR-II is shallower than that of SRR-I. Figure 6(e) shows the results for the magnetoelectric coupling parameter $\xi_{0}$. Evidently, $\xi_{0}$ of SRR-I is zero, while $\xi_{0}$ of SRR-II shows antiresonant behavior. However, this antiresonant behavior can be changed into a resonant behavior if we simply reverse the orientation of the SRR structure in the $x$ direction. All of the above retrieval results demonstrate the intrinsic differences between a normal metamaterial SRR-I and a bianisotropic metamaterial SRR-II.

\section{B. Metamaterials composed of SRRs and infinite wires}

In the previous section, we demonstrated the remarkable differences between the two metamaterials SRR-I and SRRII. Now, we will check whether those differences still remain in the metamaterials CMM-I and CMM-II, which are constructed based on SRR-I and SRR-II, respectively.

Figures 7(a)-7(d) show the amplitude and phase information of the calculated $S$ parameters for the metamaterials CMM-I and CMM-II. It can again be seen here that for the CMM-I metamaterial $S_{11}$ is equal to $S_{22}$, and $S_{12}$ is equal to $S_{21}$, since the structure is symmetric in the $x$ direction. For the CMM-II metamaterial, $S_{12}$ is equal to $S_{21}$, but neither the amplitude nor the phase of $S_{11}$ is equal to that of $S_{22}$. These characteristics of the $S$ parameters for CMM-I and CMM-II are quite similar to those of the SRR-I and SRR-II metama-
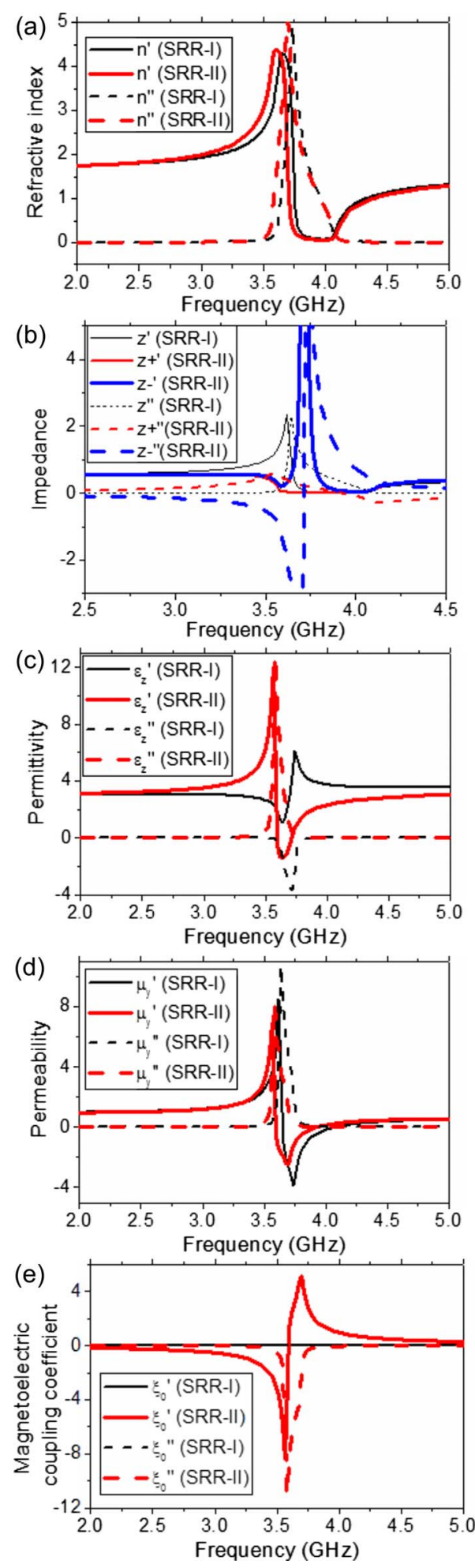

FIG. 6. (Color online) Retrieved indices (a), impedances (b), permittivity (c), permeability (d), and magnetoelectric coupling coefficient (e) for the unit cells of SRR-I and SRR-II, respectively.

terials, which we discussed in the previous section.

Figures 8(a) and 8(b) shows the retrieval results for the parameters of the refractive indices and impedances for CMM-I and CMM-II, respectively. From the results of the refractive indices, it is clearly seen that the negative index 


\section{(a)}
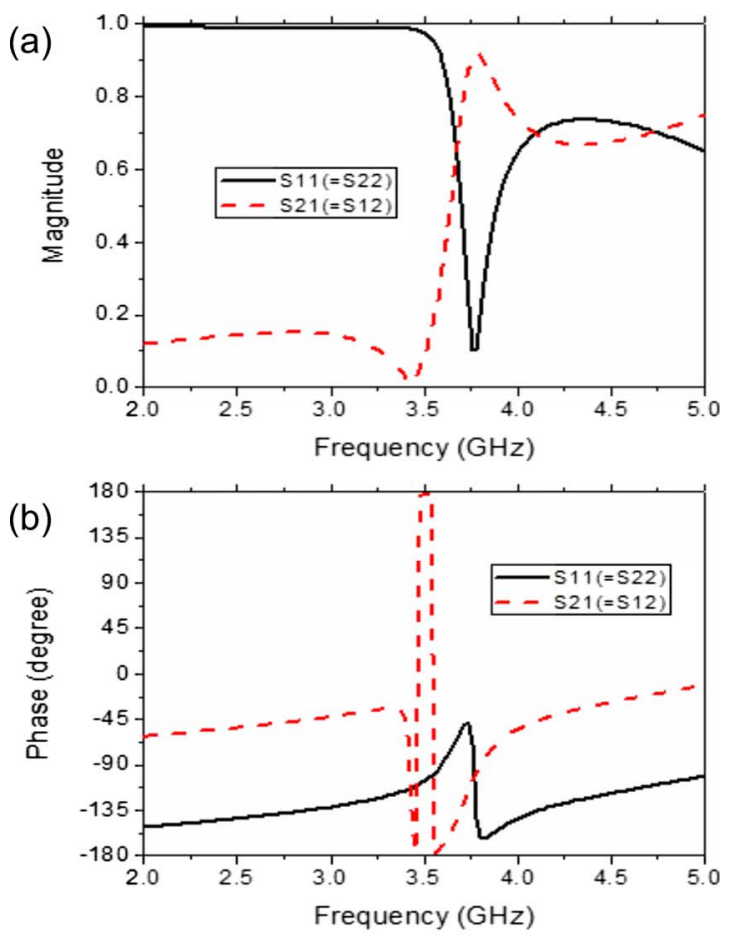

(c)
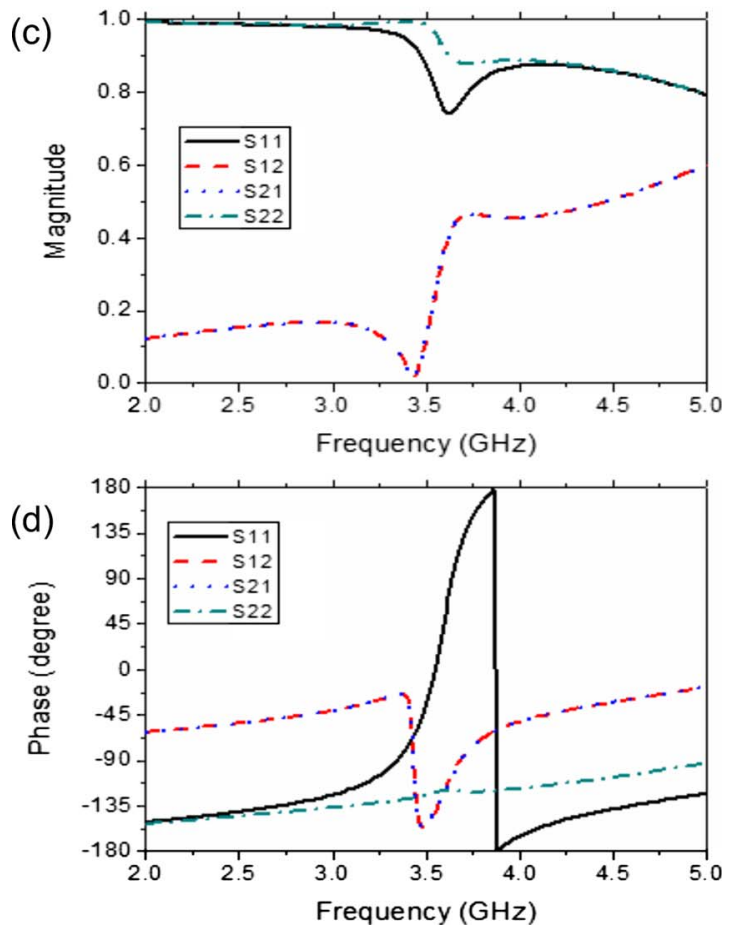

FIG. 7. (Color online) (a) Magnitude and (b) phase of the calculated $S$ parameters for the unit cell of SRR-I in Fig. 3(c). (c) Magnitude and (d) phase of the calculated $S$ parameters for the unit cell of SRR-II in Fig. 3(d).

region of CMM-II is narrower than that of CMM-I. Furthermore, the negative index region of CMM-II has a redshift compared to that of CMM-I. From the results of the impedances, it can be found that CMM-I has a wide band where the real part of the impedance is nonzero. This band actually corresponds to the pass band of CMM-I, as shown in Fig. 4. Unlike CMM-I, CMM-II has two impedances. In a frequency
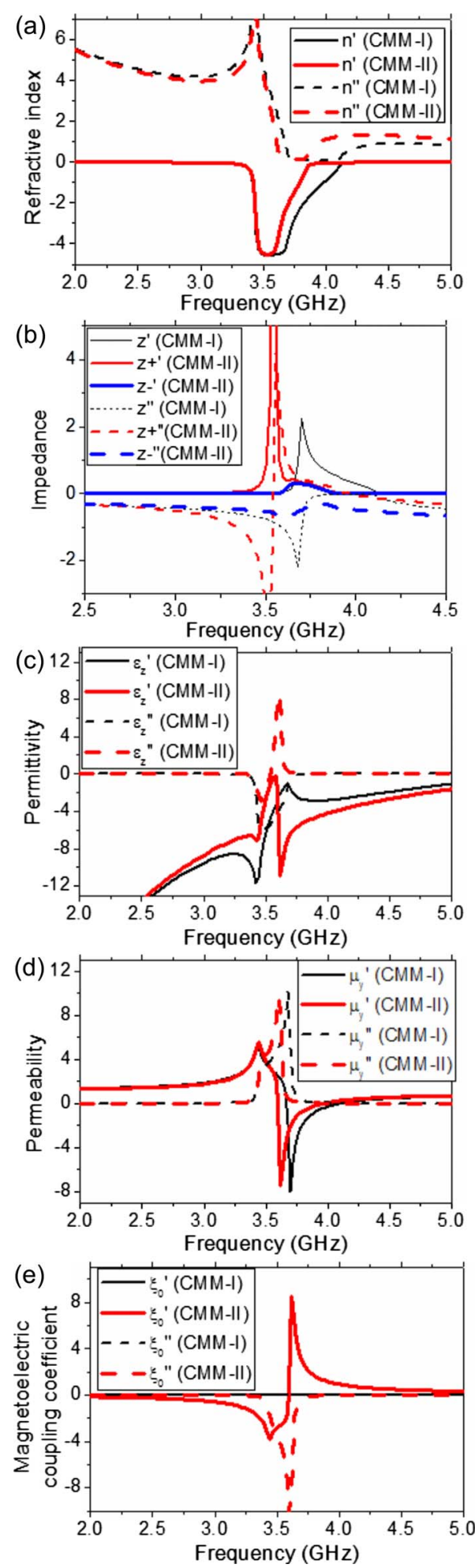

FIG. 8. (Color online) Retrieved indices (a), impedances (b), permittivity (c), permeability (d), and magnetoelectric coupling coefficient (e) for the unit cells of CMM-I and CMM-II, respectively.

range from 3.5 to $3.6 \mathrm{GHz}, z^{+\prime}$ shows a peak, while $z^{-\prime}$ shows a value of zero. This frequency range is in the stop band (cf. Fig. 4). In the frequency range from 3.6 to $3.8 \mathrm{GHz}$, both $z^{+\prime}$ and $z^{-\prime}$ have nonzero values. It is 
this frequency band that makes the passband of CMM-II (cf. Fig. 4).

The other three retrieved effective parameters of $\varepsilon_{z}, \mu_{y}$, and $\xi_{0}$ are shown in Figs. 8(c)-8(e). After comparing these results with those shown in Figs. 6(c)-6(e), it is easy to see that all the features of SRR-I and SRR-II are inherited by their corresponding composite metamaterials (CMM-I and CMM-II, respectively), except for some small deformations in the resonant regions. These small deformations come from the periodic configuration of the metamaterial [8]. It can be concluded that bianisotropy evidently exists in the metamaterial CMM-II, in which this bianisotropic characteristic intrinsically affects the constitutive parameters of the metamaterial.

\section{CONCLUSIONS}

We deduced the expressions for the calculation of $S$ parameters for a slab of a bianisotropic medium. By reversing these expressions, we obtained the analytical expressions for the retrieval of the constitutive parameters of the bianisotropic medium from the $S$ parameters. The intrinsic differences were demonstrated between a normal composite metamaterial and a bianisotropic metamaterial. Our retrieval results corroborate the conclusions of the previously published literature. Our retrieval method may be useful in future designs of composite metamaterials that utilize bianisotropy.

\section{ACKNOWLEDGMENTS}

This work is supported by the European Union under the projects EU-METAMORPHOSE, EU-PHOREMOST, EUPHOME, and EU-ECONAM, and TUBITAK under Projects No. 105E066, No. 105A005, No. 106E198, and No. 106A017. One of the authors (E.O.) also acknowledges partial support from the Turkish Academy of Sciences.
[1] V. G. Veselago, Usp. Fiz. Nauk 92, 517 (1967) [Sov. Phys. Usp. 10, 509 (1968)].

[2] J. B. Pendry, A. J. Holden, D. J. Robbins, and W. J. Stewart, J. Phys.: Condens. Matter 10, 4785 (1998).

[3] J. Pendry, A. Holten, and W. Stewart, IEEE Trans. Microwave Theory Tech. 47, 2075 (1999).

[4] D. R. Smith, W. J. Padilla, D. C. Vier, S. C. Nemat-Nasser, and S. Schultz, Phys. Rev. Lett. 84, 4184 (2000).

[5] B. I. Popa and S. A. Cummer, Phys. Rev. B 72, 165102 (2005).

[6] R. Marqués, F. Medina, and R. Rafii-El-Idrissi, Phys. Rev. B 65, 144440 (2002).

[7] D. R. Smith, S. Schultz, P. Markoš, and C. M. Soukoulis, Phys. Rev. B 65, 195104 (2002).

[8] T. Koschny, P. Markos, D. R. Smith, and C. M. Soukoulis, Phys. Rev. E 68, 065602(R) (2003).

[9] D. R. Smith, D. C. Vier, T. Koschny, and C. M. Soukoulis,
Phys. Rev. E 71, 036617 (2005).

[10] T. Koschny, P. Markoš, E. N. Economou, D. R. Smith, D. C. Vier, and C. M. Soukoulis, Phys. Rev. B 71, 245105 (2005).

[11] X. Chen, T. M. Grzegorczyk, B.-I. Wu, J. Pacheco, and J. A. Kong, Phys. Rev. E 70, 016608 (2004).

[12] N. Katsarakis, T. Koschny, M. Kafesaki, E. N. Economou, and C. M. Soukoulis, Appl. Phys. Lett. 84, 2943 (2004).

[13] S. A. Tretyakov, C. R. Simovski, and M. Hudlička, Phys. Rev. B 75, 153104 (2007).

[14] X. Chen, B.-I. Wu, J. A. Kong, and T. M. Grzegorczyk, Phys. Rev. E 71, 046610 (2005).

[15] J. A. Kong, Electromagnetic Wave Theory (EMW, Cambridge, MA, 2008).

[16] K. Aydin, K. Guven, M. Kafesaki, L. Zhang, C. M. Soukoulis, and E. Ozbay, Opt. Lett. 29, 2623 (2004).

[17] T. Koschny, M. Kafesaki, E. N. Economou, and C. M. Soukoulis, Phys. Rev. Lett. 93, 107402 (2004). 